\title{
Time-resolved temperature profile measurements in the exhaust of a single sector gas turbine combustor at realistic operating conditions
}

\author{
M. Greifenstein ${ }^{1}$ D $\cdot$ J. Heinze ${ }^{2} \cdot$ C. Willert ${ }^{2} \cdot$ L. Voigt $^{2} \cdot$ M. Zedda ${ }^{3} \cdot$ C. Richter $^{4} \cdot$ A. Dreizler ${ }^{1}$
}

Received: 13 March 2020 / Revised: 12 June 2020 / Accepted: 9 July 2020 / Published online: 20 July 2020

(c) The Author(s) 2020

\section{Abstract}

Records of the time-varying temperature profile at flight relevant operating conditions are acquired at the exit of a combustion chamber fitted with a staged, lean-burn fuel injector using high-speed laser induced fluorescence (LIF) at a sample rate of $10 \mathrm{kHz}$. Temperatures are estimated from the concentration dependent fluorescence of the hydroxyl $(\mathrm{OH})$ radical under the assumption of local equilibrium. Beyond the time-series analysis, the acquired data is correlated with simultaneously acquired $\mathrm{OH}$ chemiluminescence sampled in the primary zone near the fuel injector. These analyses reveal a strong influence from the precessing vortex core, originating in the primary zone, on oscillations in the temperature profiles measured at the exit of the combustor.

\section{Graphic abstract}

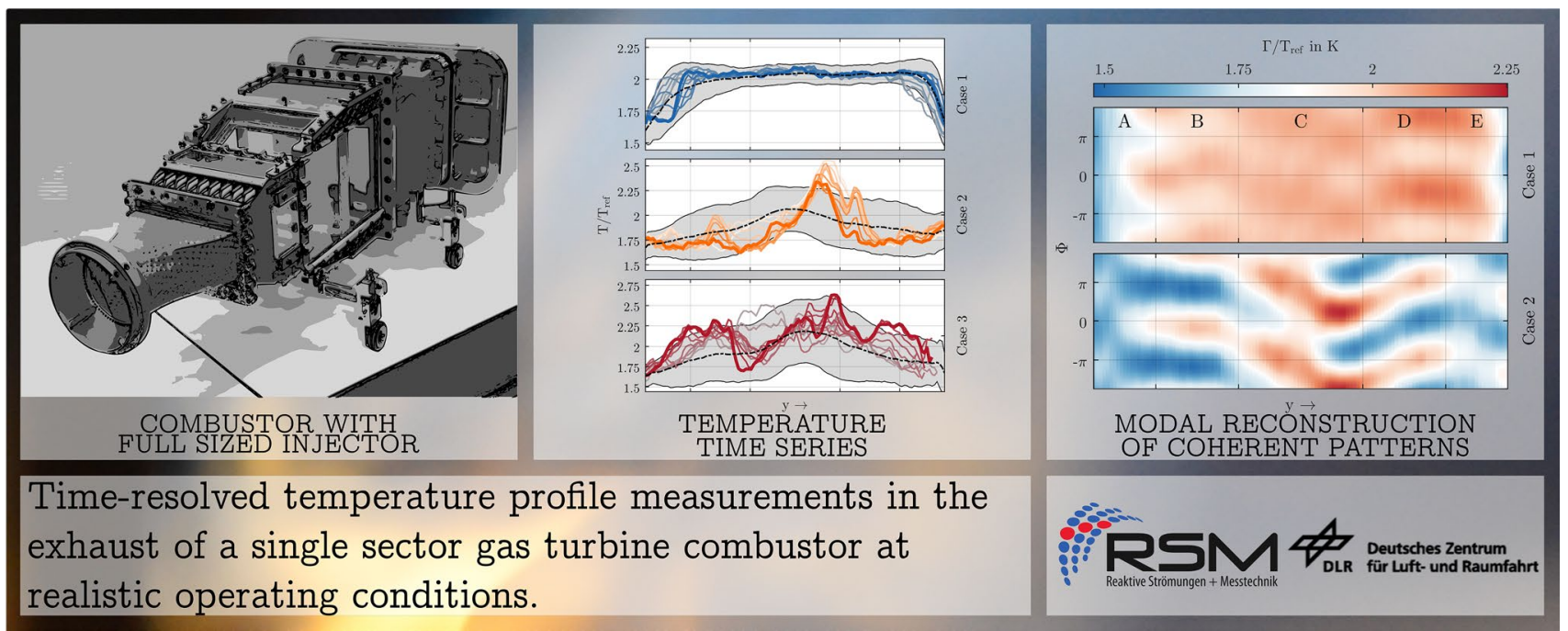

Electronic supplementary material The online version of this article (https://doi.org/10.1007/s00348-020-03011-8) contains supplementary material, which is available to authorized users.

M. Greifenstein

greifenstein@rsm.tu-darmstadt.de

$1 \quad$ Institute for Reactive Flows and Diagnostics, Technical University Darmstadt, Otto-Berndt-Str. 3, 64287 Darmstadt, Germany

2 Institute of Propulsion Technology, Deutsches Zentrum für Luft- und Raumfahrt (DLR), 51170 Köln, Germany
3 Combustion Aerothermal Methods, Rolls-Royce plc., PO Box 31, DE24 8BJ Derby, UK

4 Aeroacoustics and Noise, System Design, Rolls-Royce Deutschland Ltd \& Co KG, Eschenweg 11, Blankenfelde-Mahlow, 15827 Dahlewitz, Germany 


\section{Introduction}

To achieve the challenging ACARE Flightpath 2050 targets for the reduction of $\mathrm{CO}_{2}, \mathrm{NO}_{x}$ and noise emissions from of aeroengines, the combustion process will need to be further optimised. Improved high fidelity methods and low order models are needed for this optimisation. Suitable component level testing is realized with dedicated test facilities that aim to reproduce the flight-relevant conditions of the propulsion system. Detailed measurement methods, that can cope with the challenging high temperature pressurized environment, are key in the acquisition of suitable validation data for method validation. Such detailed modern measurement technologies provide further insight into the underlying complex aero-thermal processes. In this context, non-contact and non-intrusive optical diagnostics in particular play a crucial role in gaining insights to physical phenomena and providing important validation data.

This investigation presents an accurate and precise technique for spatially and temporally resolved temperature measurements that allows to develop-in conjunction with other techniques - an improved understanding of the physics involved in the generation and propagation of indirect noise in low emission combustors. The noise generated by the combustion chamber includes both the direct noise and indirect noise. The former is generated by pressure waves related to the unsteadiness in the rate of heat release. The latter, also sometimes referred to as entropy noise is mainly generated by entropy waves, that is, non-uniform temperature patches that are accelerated through the nozzle guide vanes (NGVs) at the combustor exit and interact with the blades of the following turbine stages. For more information regarding entropy noise, the reader is referred to the literature, e.g. Ihme (2017); Morgans and Duran (2016); Bake et al. (2009); Bauerheim et al. (2016); Hield et al. (2009). Beyond the noise generation through spatio-temporal variation of temperature, knowledge of the unsteady temperature distribution at the exit of the combustion chamber is of high value for reliable design of cooling strategies of NGVs and the performance of the downstream high-pressure turbine stages (Doll et al. 2018).

This publication focuses on the development, application and processing of the measurement technique as well as documenting recent efforts to provide reference data for a low emissions aero-engine combustion chamber operated at engine relevant conditions beyond classical statistically independent sampling (Meier et al. 2015). In the first part of this article the fully optically accessible singlesector combustor OCORE 2 is presented. Subsequently, the measurement strategy and the experimental setup for spatially and temporally resolved temperature measurements at the combustor exit is described. The acquired data is correlated with time series of $\mathrm{OH}^{*}$ chemiluminescence measurements in the primary zone as an indicator for heat release. To the authors' knowledge the measurements presented herein are the first of its kind obtained in a combustion test facility operated at operating conditions that are representative of the conditions found in an aeroengine in flight.

\section{Experimental setup}

\subsection{Single sector combustor}

The optically accessible single-sector combustor used for the present investigation is schematically outlined in Fig. 1, top. It consists of the actual combustion chamber liner, named OCORE 2, which is housed inside a pressure vessel of the high pressure testing facility HBK1 located at the DLR Institute of Technology in Cologne. A photograph of the combustion chamber liner is shown in Fig. 1, bottom. The liner is made of Inconel-a nickel-chromium
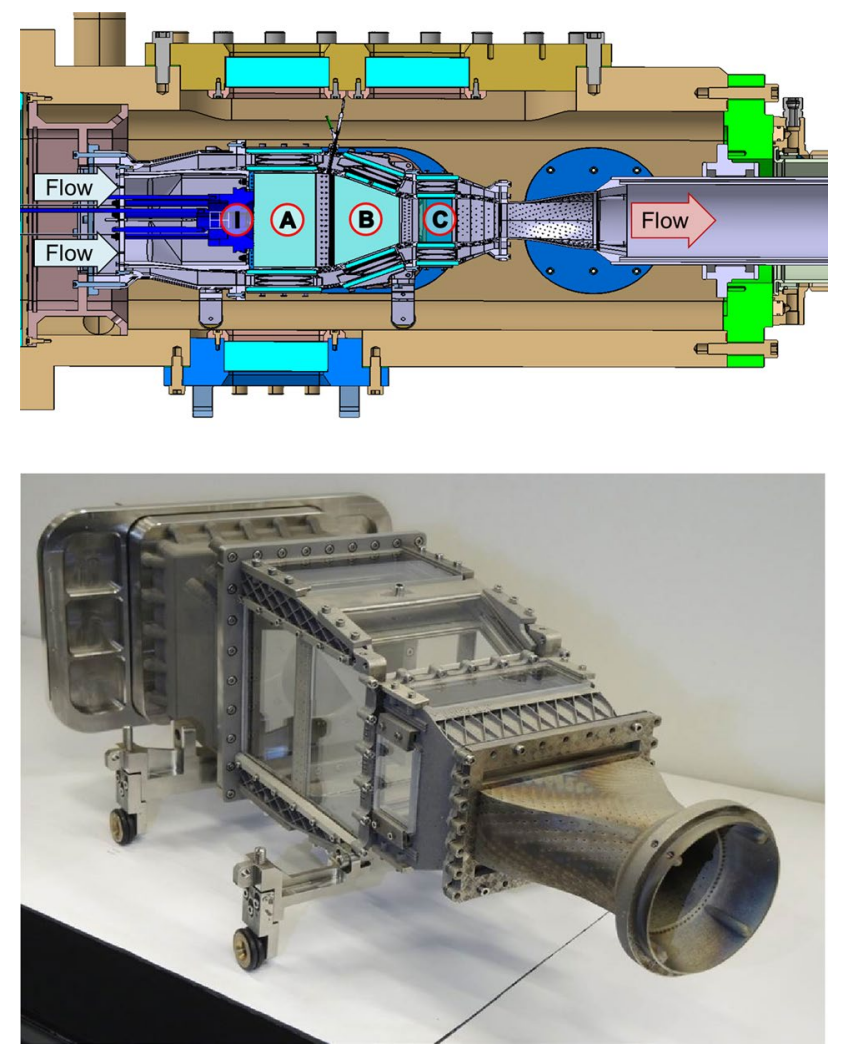

Fig. 1 Top: CAD drawing of the liner inside the pressure vessel. The fuel injector is located at (I), primary combustion zone (a), converging zone (b) and combustor exit (c). Bottom: OCORE 2 combustion chamber liner manufactured through 3-D printing 
based superalloy—using laser based additive manufacturing (i.e. direct laser deposition, DLD). During operation of the combustor, preheated, pressurized air is introduced from a plenum located to the left of the combustion chamber, supplying both the fuel injector (I) as well as the liner cooling through separate ducts. This allows the preheated air along with the hot combustion products to be confined to the liner while the exterior flow inside the pressurized test section remains at ambient conditions. Thick, uncooled quartz glass windows provide optical access into the test section. Optical access to the interior combustion zones is achieved by additional thin windows on the liner itself. These windows are only exposed to a minimal pressure differential and are partially air film cooled using the preheated supply air.

The liner of the combustion chamber shown in Fig. 1, top, mimics the geometry of the aeroengine counterpart featuring a primary zone (A), a converging zone (B) and an exit zone (C). The flow downstream of the two-staged swirled fuel injector (I) recirculates within zones (A) and (B) which has a stabilizing effect on the combustion (Hassa et al. 2017). In the convergent zone the flow accelerates before leaving the combustor. In the engine counterpart, zone (C) coincides with the position of the nozzle guide vanes (NGV) upstream of the first high pressure turbine stage. Within this study, three operating conditions at the same elevated pressure $\left(p_{30}>10\right.$ bar) and combustor inlet temperature were investigated, see table 1 . The operating conditions vary in fuel split (FS, ratio between fuel content in pilot and main) and global air-fuel-ratio (AFR). Due to the combustion air flow split of the injector, at the given fuel split for Case 1, main and pilot operate at the same nominal AFR, that is, there is no fuel stratification. For all measured cases, the mixture is globally lean. Pilot and main stage operate in a premixed or partially premixed combustion mode. All measurements were performed when the combustor was in thermal equilibrium to ensure no influence from varying wall heat flux during the acquisition.

\subsection{Diagnostics}

The underlying concept of the in-situ high-speed temperature measurement technique used in this experiment is to determine optically the absolute local concentration of the $\mathrm{OH}$ radical as a highly sensitive temperature indicator molecule, whose concentration grows nearly exponentially with

Table 1 Operating conditions

\begin{tabular}{llll}
\hline Name & FS & AFR & Comment \\
\hline Case 1 & Low & Low & AFR balanced between main and pilot \\
Case 2 & Intermediate & High & AFR different between main and pilot \\
Case 3 & High & High & Pilot-only operation \\
\hline
\end{tabular}

respect to temperature (Heinze et al. 2011; Morley 2005). The applicability of this method is restricted to areas with a lean mixture $(\lambda \geq 1.1$ or $\Phi \leq 0.9)$ and chemical equilibrium (Heinze et al. 2011). Under these conditions, the OH concentration is approximately independent of the equivalence ratio $\Phi$ and can be assigned unambiguously to temperature. Under the given boundary conditions (i.e. elevated pressure, high preheating temperatures), the chemical relaxation time of the $\mathrm{OH}$ super-equilibrium concentration of $<0.1 \mathrm{~ms}$ (Heinze et al. 2011) is substantially shorter than the $\mathrm{OH}$ transit time of $4-7 \mathrm{~ms}$ (see Sect. 3.5) between the primary zone and the measurement location at the combustor exit. The equivalence ratios of all examined operating conditions are well below the upper validity limit of $\Phi \leq 0.9$.

The local $\mathrm{OH}$ distribution $[\mathrm{OH}(x)]$ along the direction $x$ of the exciting laser can be deduced from simultaneous measurements of the spatially resolved OH (P)LIF intensity $I_{\mathrm{LIF}}(x)$ and the integral line-of-site resonant absorption $A$ of the exciting laser beam (Pixner et al. 2000; Arnold et al. 1997; Heinze et al. 2011). Therefore, the OH LIF image represents the derivative of the local laser intensity along the beam. Since the final intensity value is given by the absorption measurement, the local laser intensity can be easily calculated by integration. By normalizing to the local laser intensity, the LIF image is absorption corrected to $I_{\mathrm{LIF}}^{*}$ and then calibrated using the relationship between Einstein B coefficient and absorption. All spectroscopic quantities are well known for the $\mathrm{OH}$ molecule (Luque and Crosley 1999). Equation 1 describes how the $\mathrm{OH}$ concentration is deduced from measured quantities and spectroscopic data.

$[\mathrm{OH}(x)] \cong I_{\mathrm{LIF}}^{*}(x) \frac{A}{\int I_{\mathrm{LIF}}(x) \mathrm{d} x} \frac{1}{g_{0} h \nu_{j i} B_{j i} D_{i} f_{i}^{B}}$

Here, $g_{0}$ denotes the spectral overlap of the absorption line and the initial laser line, while $v_{j i}$ is the excitation frequency from state $i$ to state $j$ with Einstein B coefficient $\mathrm{B}_{j i}$, degeneracy factor $D_{i}$ and Boltzmann factor $f_{i}^{\mathrm{B}}$. Implicitly, the quantities $f_{i}^{\mathrm{B}}, g_{0}$ and $I_{\mathrm{LIF}}^{*}$ depend on the local temperature and/or gas composition. A proper choice for the excitation transition $j \leftarrow i$ is necessary to minimize this influence. Under the given conditions, the $Q 1(9.5)+Q 2(7.5)$ line pair of the $\mathrm{OH} A^{2} \Sigma \leftarrow \mathrm{OH} X^{2} \Pi\left(v^{\prime}=1 \leftarrow v^{\prime \prime}=0\right)$ transition at $35,210.42 \mathrm{~cm}^{-1}$ provides some important features: The combined Boltzmann factor $f_{i}^{\mathrm{B}}(T)$ of the double line varies only by $10 \%$ from $1200 \mathrm{~K}$ up to $2400 \mathrm{~K}$. Additionally, at elevated pressures, the double line is the strongest absorption line, allowing high signal to noise ratios, and has an almost tophat like line shape, simplifying the experimental absorption measurement while reducing numerical uncertainties in the calculation of the spectral line overlap.

Assuming chemical equilibrium, the $[\mathrm{OH}]-T$ correlation is calculated from 0D chemical simulations (Morley 2005) 
which is subsequently used to map the spatially resolved $\mathrm{OH}$ concentration to a temperature field. Compared to the more commonly used OH LIF 2-line thermometry (GiezendannerThoben et al. 2005; Devillers et al. 2008) the main advantages of this approach are a 5-10 times higher temperature sensitivity due to the near-exponential growth of $[\mathrm{OH}]$ with temperature and a substantially higher signal-to-noise ratio (Heinze et al. 2011). However, the same fact leads to a reduced lower detection limit of typically $1400 \mathrm{~K}$ due to the limited dynamic range for a $2 \mathrm{D}$ detection system in a combustion chamber caused by false signal light (Doll et al. 2017). Due to the smaller beam dimension in the presented experiment, a substantially higher dynamic range-compared to previous experiments at $10 \mathrm{~Hz}$ (Doll et al. 2017) of about 1000 was achieved which effectively improved the lower detection limit to $1200 \mathrm{~K}$. The extension of the temperature range with respect to the lower limit is particularly noteworthy considering the limited available pulse energy of the frequency doubled high-speed dye laser system.

The excellent $\mathrm{S} / \mathrm{N}$ ratio and additionally the fact that the experimental effort is significantly reduced compared to 2-line LIF thermometry enable a precise, yet cost effective temperature measurement technique in this high-speed experiment under the harsh conditions of a gas turbine test rig.

For a more detailed description of the OH-thermometry method, the reader is referred to Heinze et al. (2011).

Optical setup $\mathrm{OH}^{*}$ chemiluminescence in the primary zone was captured using a photomultiplier tube (PMT) (Hamamatsu, 1P28) equipped with UV lens (UV Nikkor $105 \mathrm{~mm})$ at $f / 11$. A band pass filter in front of the lens with a transmission range from $300-325 \mathrm{~nm}$ (Laser Components) was used to spectrally separate the $\mathrm{OH}^{*}$ signal.

Using a current-to-voltage converter (Hamamatsu C7319) as preamplifier, the photomultiplier signal was digitized (National Instruments, NI 9215) and recorded as time series with a sampling rate of $50 \mathrm{kHz}$ along with the camera trigger for further data evaluation.

For the combined LIF/absorption measurements, a high speed dye laser system (Sirah, Allegro) with Rhodamin 6G dissolved in ethanol was optically pumped with a diode-pumped solid state Nd:YAG laser (Edgewave, Innoslab) at an average optical power of $80 \mathrm{~W}$. To excite the $A^{2} \Sigma \leftarrow \mathrm{OH} X^{2} \Pi\left(v^{\prime}=1 \leftarrow v^{\prime \prime}=0\right)$ transition of $\mathrm{OH}$, the fundamental wavelength of the dye laser was tuned to $17,605.21 \mathrm{~cm}^{-1}$, corresponding to the center frequency of the Q1(9.5)+Q2(7.5) line pair at 35,210.42 $\mathrm{cm}^{-1}$. Frequency doubling of the fundamental wavelength was achieved with a pair of temperature stabilized BBO crystals in a compensator configuration. A fiber coupled high precision wavemeter (High Finesse, WS Ultimate 30) was used to monitor the fundamental wavelength and linewidth. To ensure accurate measurements of the central frequency, the wavemeter was

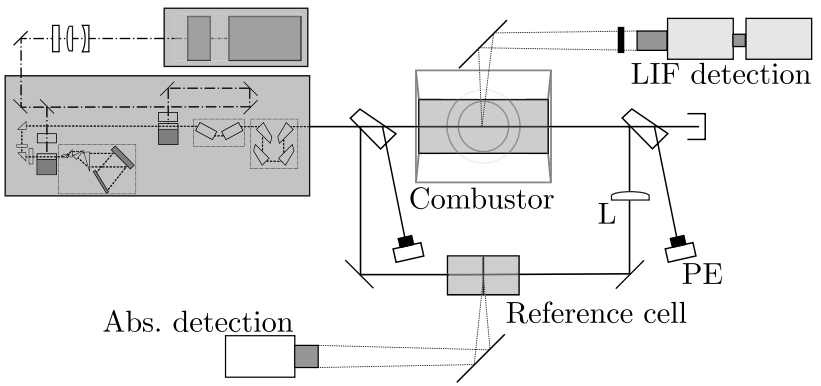

Fig. 2 Optical setup for high-speed OH-LIF. PE: pyroelectric energy meter; L: 4f-imaging lens

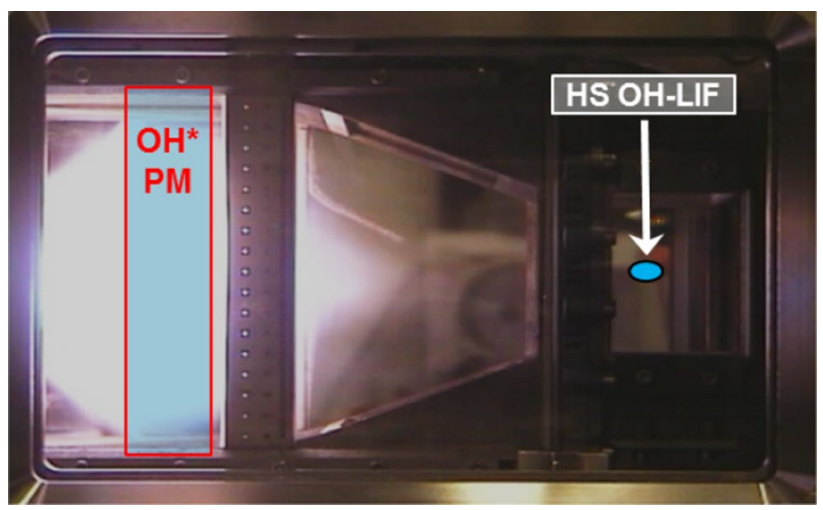

Fig. 3 Measurement locations for high-speed $\mathrm{OH}-\mathrm{LIF}$ and $\mathrm{OH}^{*}$ chemiluminescence

calibrated at regular intervals using a temperature stabilized single-mode He-Ne laser. Resulting radiation in the UV was separated from the fundamental using dichroic mirrors instead of Pellin-Broca prisms to maximize available pulse energy. The maximum available pulse energy was $380 \mu \mathrm{J}$ at a linewidth of $0.15 \mathrm{~cm}^{-1}$ (calculated from $\sqrt{2}$ times the fundamental line width).

$\mathrm{OH}$ fluorescence was detected using a combination of a high speed CMOS camera (Photron, SA-X2), a high speed lens-coupled intensifier (LaVision, HS-IRO) equipped with a $100 \mathrm{~mm}$ UV lens at $\mathrm{f} / 2.8$ (Cerco, 2178). A narrow-band filter (AHF Analysentechnik, $\mathrm{T} \geq 90 \%$ at $312-322 \mathrm{~nm}$ ) was used to spectrally isolate the $(1,1)$ vibrational band of $\mathrm{OH}$, minimizing influences from superimposed kerosene LIF as well as reabsorption of signal photons (Sadanandan et al. 2012). Additionally, an IR-blocking filter (ITOS, UG11IRB, $2 \mathrm{~mm}$ ) was used to suppress contributions from thermal radiation. Figures 2 and 3 show a sketch of the optical setup and the location of the measurement locations within the combustor. To distinguish the desired quantity absorption from other loss mechanisms (e.g. reflections on windows), absorption is determined using an on-off-resonancehopping approach. In this scheme, absorption is derived from the energy transfer function ratio of on-resonant and 
off-resonant measurements, see Eq. (2), where $A$ denotes the integral absorption and $E_{i}$ a measure of the pulse energy.

$A=1-\frac{E_{\text {on,out }} / E_{\text {on,in }}}{\overline{E_{\text {off,out }}} / \overline{E_{\text {off,in }}}}$

Due to the nature of this scheme, on- and off-resonant measurements cannot be captured simultaneously. To account for potential time-varying losses (e.g. window fouling), offresonant measurements are acquired in short succession for every on-resonant sequence. For off-resonant measurements, the laser was tuned about $15 \mathrm{~cm}^{-1}$ towards higher wavenumbers. Two wedged plate beamsplitters (Thorlabs, BSF2550, wedge angle $5^{\circ}$ ) were used to redirect a portion of the beam to the energy detection systems. This allows to generate two spatially well separated reflexes that are applied to two independent energy detection systems.

A pair of pyroelectric energy meters (Coherent, $\mathrm{J}-10-\mathrm{MT}-10 \mathrm{kHz}$ ) is used to monitor the absolute pulse energy to ensure a properly working laser system in the harsh environment. However, the signal-to-noise ratio (SNR) is very limited due to the detectors' strong sensitivity to vibrations and high sound pressure levels in the vicinity of the rig.

As a second measure of the pulse energies, an optically accessible reference cell filled with a mixture of de-ionized water, ethanol and Rhodamin 6G was used. The dye concentration in the reference cell was optimized for a high signal while keeping absorption effects small. To minimize degrading of the dye, a modified cell with a high through-put circulator with external water cooling (Sirah, HPPC-DC-R20) was used. A second high speed camera (Photron, SA-X2) equipped with a $180 \mathrm{~mm}$ lens at f/5.6 (Sigma, AF 2.8/180 EX DG OS HSM) was used to detect the red-shifted fluorescence signal. To minimize beam-steering, a lens in a $4 f$ arrangement $(f=200 \mathrm{~mm}$ ) imaged the central part of the combustor onto the detection systems.

For every operating condition, six statistically independent runs with a length of 28000 on-resonant samples (i.e. $2.8 \mathrm{~s})$ were acquired. Each set was followed by 1000 offresonant samples with short delay $(\approx 10 \mathrm{~s}$, limited by the time to accurately tune the laser).

Processing To derive temperature information from the combined LIF/absorption measurements, several processing steps are necessary. At first, a background subtraction is performed for both detection systems. The background corrected LIF images are binned in cross-beam direction over the entire width of the signal. Additionally, a 4 px spatial binning in beam-wise direction is used to reduce noise while preserving the optical resolution of the intensifier. The integral absorption is derived by spatially integrating the input and output reflexes in the reference cell image and calculating the respective ratios (see Eq. 2). The inferred absorption is used as a boundary condition for the absorption correction of the LIF image, i.e. the local laser intensity is reconstructed by accounting for the absorption of every single pixel. Spatially resolved absorption is derived by distributing the integral absorption proportional to the LIF signal. The calibration constant from absorption to $[\mathrm{OH}]$ is inferred from the spectral overlap of a spectroscopic simulation (Luque and Crosley 1999) for every operating condition and a Gaussian laser lineshape with the measured linewidth. Correlations of $[\mathrm{OH}]$ and temperature are obtained from 0D-simulations using GASEQ (Morley 2005).

\section{Results and discussion}

The aim of this publication is to show the viability of timeresolved temperature measurements in a single sector gas turbine combustor. Hence, the following sections focus on extracting information which would not be available using conventional low-speed techniques. At first, a discussion about the accuracy and precision of the method is presented. Time series and averaged temperatures give an impression of the differences in terms of operating conditions to guide the reader through the following frequency and correlation analyses.

\subsection{Accuracy and precision}

To assess the quality of the derived temperature profiles, one has to consider the accuracy and precision of the individual measurement systems. According to Eq. (1), the integral absorption $A$ is proportional to [OH $(x)]$, hence accuracy and precision of the absorption measurement transfers directly to the uncertainties of the inferred local total number density. Due to the ratio of local fluorescence and integral fluorescence along the beam, the accuracy of the LIF signal cancels for single-shot evaluation while the spatial precision influences the spatial distribution of $[\mathrm{OH}]$.

Absorption According to Eq. (2), the absorption is inferred from the ratio of signal counts. Highspeed CMOS cameras are known to exhibit a spatially inhomogeneous non-linear response to irradiance. In a thermally stable state, this non-linearity can be corrected using measurements at different irradiances, e.g. with an integrating sphere (Weber et al. 2011). Due to strong temperature fluctuations in the facility, this non-linear correction cannot be performed reliably. Instead, an estimation of the accuracy error bounds is performed by comparing the ratio of grey values acquired from integrating sphere data without non-linearity correction for comparable count levels as found in the experiment with a linear fit, see Fig. 4. Systematically, lower absorption values are overestimated while higher absorption values are underestimated. With respect to the temperature measurement, this will lead to a 


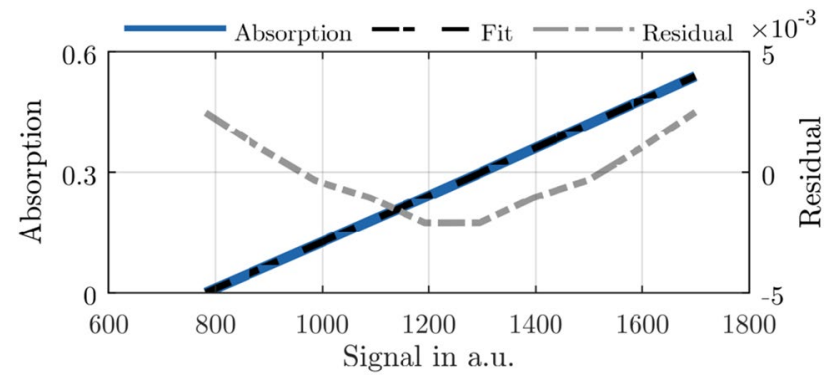

Fig. 4 Estimation of the accuracy of the absorption measurement

compression of the measured range. However, as the absolute residual is well below $1 \%$, this effect can be neglected.

As a measure of the precision, the temporal relative standard deviation for the in-out-ratios of the off-resonant measurements is considered ( $\left.E_{\text {off,out }} / E_{\text {off,in }}\right)$. This includes operating-condition dependent effects (e.g. beam steering, vibrations, sound pressure levels) and all detector uncertainties combined.

With values of $\approx 1.5 \%$, Case 1 exhibits the lowest relative standard deviation, probably due to comparably low temperature fluctuations within the measurement area which lead to lower variations in refractive index, see Sect. 3.2. Case 2 and 3 show deviations in the order of 5\% due to higher temperature fluctuations caused by increasing fuel split.

LIF Estimating the precision of the LIF detection system is not as straightforward as it is for the absorption since no steady-signal is available in-situ. Instead, values from the literature for the spatial standard deviation in the order of 5\% are considered (Weber et al. 2011). The cited investigation used an integrating sphere, i.e. a homogeneous illumination at different irradiances of the entire active area of the intensifier. This is a worst case scenario, as intensifier depletion and non-linearity effects strongly depend on the area of illumination. In the present study, less than $10 \%$ of the active area is used.

Spectroscopy Additionally, the spectroscopic constants add uncertainties to the evaluation. These are $5 \%$ for the Einstein B coefficient, $8 \%$ for the Boltzmann factor due to its temperature dependence and $10 \%$ for the spectral laser line overlap (Heinze et al. 2011).

Effect of uncertainties on temperature evaluation Following Gaussian error propagation, the combined uncertainties for the local total number density $[\mathrm{OH}(x)]$ is $\approx 14 \%$. In the temperature range relevant for this study, the total $\mathrm{OH}$ number density approximately doubles every $100 \mathrm{~K}$. For a temperature of $1600 \mathrm{~K}$, the resulting temperate error bar spans $\pm 20 \mathrm{~K}$.

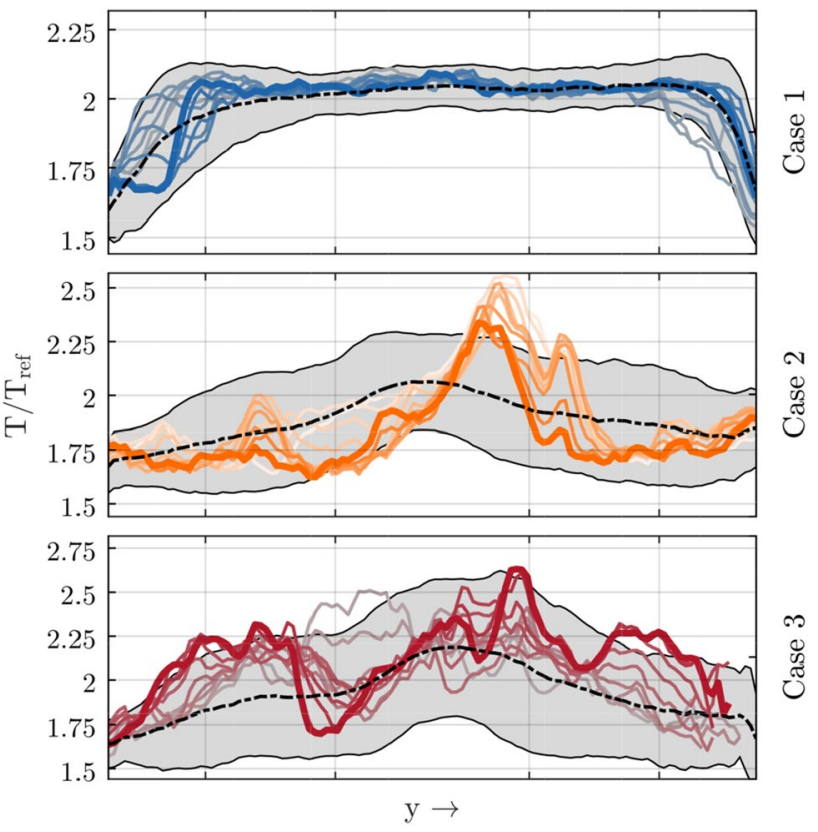

Fig. 5 Line plots of 10 successive temperature measurements with $100 \mu$ s time separation. Increasing color saturation indicates temporal evolution. Grey shaded area represents mean plus/minus one standard deviation. Mean temperature profile from all samples represented by dash-dotted black line. An animated version of this Figure showing a $90 \mathrm{~ms}$ time series is available online

\subsection{Temperature profiles}

Figure 5 shows an exemplary timeseries of $1 \mathrm{~ms}$ in color over a shaded area representing the mean temperature profile plus/minus one standard deviation for all operating conditions measured in this study. Throughout this publication, all temperatures are non-dimensionalized with a constant reference temperature. As expected, with increasing fuel split and the associated decrease in local AFR, peak temperature level increases as well as the overall inhomogeneity and fluctuations along the measured profile. The temperature wake of the pilot can be clearly identified in the Figure for Case 2 and 3 in the central region. Peak temperatures for Case 3 are significantly higher than for Case 1 . In the central region, standard deviation spans from $\approx 2 \%$ for Case 1 to $\approx 11 \%$ for Case 3 . The burner exhibits a slight case dependent asymmetry with respect to the central axis. For Case 1 and 2, temperatures in the right half of the plot are systematically higher compared to the left side while Case 3 is mostly symmetrical. This is an indication that the main stage is not strictly symmetrical with respect to the central axis. The temporal evolution of 10 successive temperature measurements indicate the temporal coherence and give an impression of the dynamics of the underlying phenomena. Case 1 exhibits the strongest fluctuations close to the walls, where the temperature close to the left wall changes by $\approx 20 \%$ with respect to 
the mean within $1 \mathrm{~ms}$. Amplitudes for Case 2 are in a similar order, with a shift to higher fluctuations in the center. As expected from the high standard deviations, Case 3 shows the strongest dynamics in terms of temporal fluctuations. Within $1 \mathrm{~ms}$, temperature changes by up to $38 \%$ near the central axis of the burner. The shift of high standard deviations from near-wall (Case 1) to the central region (Case 2 and 3) can be visualized in temperature probability density functions (PDF), shown in Fig. 6. Case 1 exhibits a bi-modal distribution close to the walls (regions A and E) for reasons to be discussed later. Peaks in the "hot" area of the near-wall PDFs correspond to the very narrow peak temperature in the central region $(\mathrm{C})$. Overall, except effects close to the walls, this operating condition is fairly symmetrical with the narrowest PDFs in the center. Case 2 shows an opposing trend. Temperature PDFs tend to be lower and broader in the central region (C) due to the increasing effect of the highly fluctuating pilot. Slight asymmetries with respect to the center line can be observed in the near-wall PDFs (A,E). In the temperature wake of the main stage (B,D), PDFs are

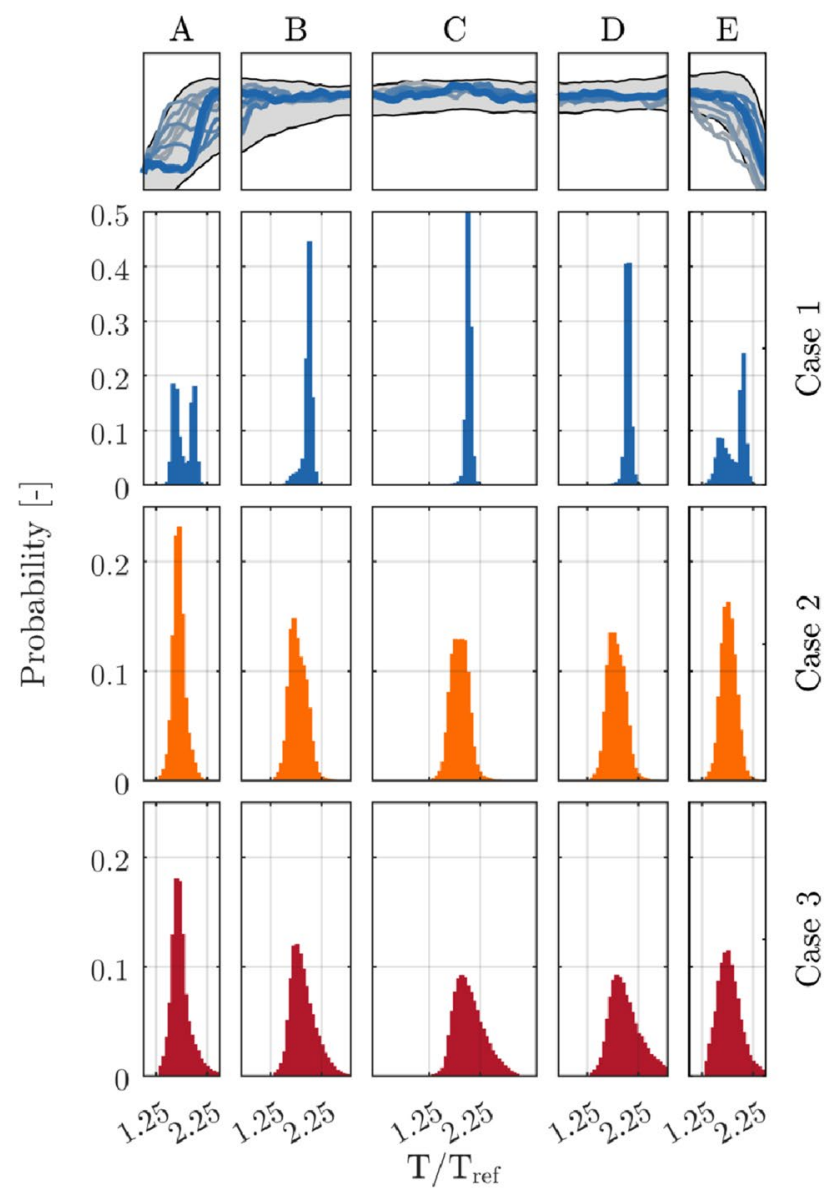

Fig. 6 Temperature PDFs for a single run of all cases. Top row shows timeseries for Case 1 as an orientation only. Capital letters denote regions of interest used throughout this paper skewed with a smaller gradient towards higher temperatures. The trend towards stronger skewness and asymmetry develops with increasing fuel split. Case 3 shows highly skewed distributions towards higher temperatures in all regions due to the higher local equivalence ratio upstream. As the pilot stage is not surrounded by a reacting main stage, the very high fluctuations reach further into areas closer to the wall. The trend to broader PDFs from left to right $(\mathrm{A} \rightarrow \mathrm{E})$ becomes more evident. Overall, the temperature PDFs agree well with the expectations about homogeneity, spatio-temporal fluctuations and global temperature level. Due to the lower AFR of Case 1, peaks in the PDFs are centered at a higher temperature compared to Case 2 and 3 .

\subsection{Frequency analysis}

One-dimensional time-resolved measurements allow the analysis in frequency domain for every point along the measured profile. Figure 7 shows Fourier transforms (FT) of the temperature fluctuation time series $\mathcal{T}_{i}^{+}$, see Eq. (3), where $i$ denotes a pixel along the dimensionless coordinate $y, \mathcal{F}$ the FT operator and $\bar{T}_{i}$ the temporal mean for pixel $i$.

$\mathcal{T}_{i}^{\prime}=\mathcal{F}\left(T_{i}^{\prime}\right)=\mathcal{F}\left(T_{i}-\bar{T}_{i}\right)$

For Case 1, a strong interaction with the combustor walls is observed $(\mathrm{A}, \mathrm{E})$ at $\approx 100 \mathrm{~Hz}$. This was already indicated

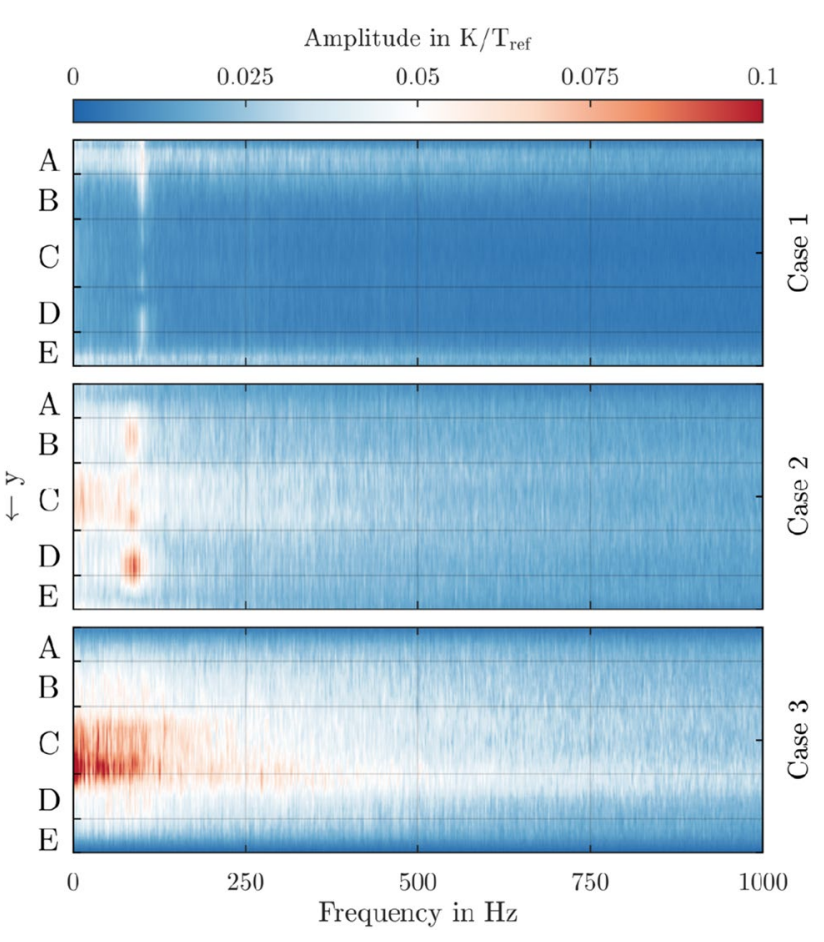

Fig. 7 Spatially resolved frequency spectra for the selected cases 
in the time series in Fig. 5. The influence decays towards the center but still impacts the wake of the main stage (B,D). Increasing fuel split for Case 2 and 3 correlates with increasing broadband noise in the central region of the combustor (C). Additionally, Case 2 exhibits a strong oscillation around $\approx 92 \mathrm{~Hz}$ in the wake of the main (B,D). The pilot-only Case 3 is free from any prominent frequency peaks.

Interestingly, Case 1 and 2 show very similar characteristic frequencies, which have a fundamentally different modal appearance. Figure 8 shows the reconstructed modes at the respective maximum amplitudes. The reconstruction follows Eq. (4, where the reconstruction $\Gamma$ for a selected frequency $\omega$ is computed using the amplitude $a_{i}$ and initial phase $\Phi_{0, i}$ as determined from the complex power spectrum, for every pixel. As the FT is based on temperature fluctuations, the temporal mean $\bar{T}_{i}$ is added after the the reconstruction of the oscillation.

$$
\begin{aligned}
a_{i}(\omega) & =\left|\mathcal{T}_{i}^{+}\right|=\sqrt{\operatorname{Re}\left(\mathcal{T}_{i}^{+}(\omega)\right)^{2}+\operatorname{Im}\left(\mathcal{T}_{i}^{+}(\omega)\right)^{2}} \\
\Phi_{0, i}(\omega) & =\arctan \left(\frac{\operatorname{Im}\left(\mathcal{T}_{i}^{+}(\omega)\right)}{\operatorname{Re}\left(\mathcal{T}_{i}^{+}(\omega)\right)}\right) \\
\Gamma(\Phi, \omega) & =a_{i}(\omega) \cos \left(\Phi+\Phi_{0, i}(\omega)\right)+\bar{T}_{i}, \Phi \in[-2 \pi ; 2 \pi]
\end{aligned}
$$

This modal analysis reveals that the sudden drop in temperature seen in Fig. 5 close to the walls for Case 1 is indeed a periodic event with a frequency of $\approx 100 \mathrm{~Hz}$. For Case 2 , the characteristic frequency is caused by an anti-symmetrical oscillation of the left and right half of the main stage. Most likely, this anti-symmetric oscillation is related to a sectional cut of the precessing vortex core (PVC) which gives rise to the characteristic $\mathrm{V}$-shaped pattern for the modal reconstruction of Case 2. High-speed flow field measurements using

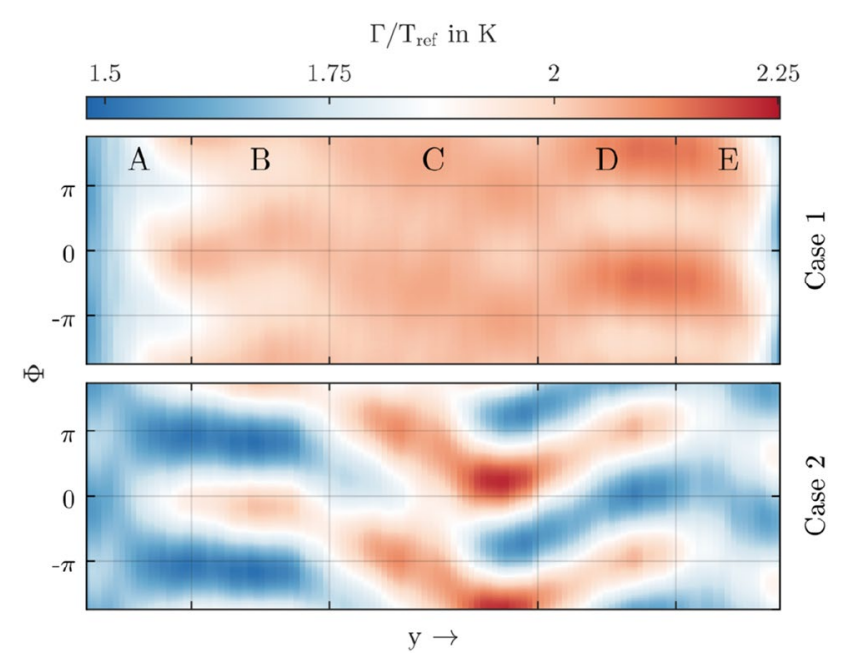

Fig. 8 Reconstructed mode for characteristic frequencies for Case 1 and 2
PIV in the exit duct at identical operating conditions as in this paper support the hypothesis of a present PVC (Willert et al. 2019). In fact, this pattern is also imprinted in the reconstruction of Case 1, albeit with a very low amplitude of only a few $\mathrm{K}$ due to the fact that the main and pilot stage operate at (nominally) the same AFR. However, the temperature drop in region A coincides with the "cold" side of the PVC approaching the wall where it further looses enthalpy until a point where reaction zones are quenched, leading to the sudden drop in temperature and potentially fuel slip. The asymmetry found for the main stage in the mean temperature profiles is also reflected in the modal reconstruction as well as the frequency spectra.

\subsection{Integral timescales}

Integral timescales $\Lambda$ give a rough estimate of the duration of temperature correlation. With the available time-resolved data, this can be computed directly from the variance normalized integration from temporal auto-covariances $C_{T}$ for every pixel $i$ along the measured temperature profile. In Eq. (5), $N$ denotes the number of available datapoints, $\sigma_{i}^{2}$ the variance of the timeseries at pixel $i, m$ the temporal lag for the auto-covariance in units of the sampling interval $\Delta t$ and $\tau$ the temporal lag in ms.

$$
\begin{aligned}
\Lambda_{i} & =\frac{1}{\sigma_{i}^{2}} \sum_{k=0}^{N} C_{T}(m) \Delta t \\
C_{T}(m) & =\sum_{n=0}^{N-m-1}\left(T_{n+m}-\frac{1}{N} \sum_{k=0}^{N-1} T_{k}\right)^{2} \\
\tau & =m \Delta t=\frac{m}{10} \mathrm{~ms}
\end{aligned}
$$

Figure 9 shows the spatially resolved variance normalized auto-covariances for the selected cases. The auto-covariances were computed for every run individually and then averaged over the number of runs. Similar to the frequency spectra, the auto-covariance exhibits significant spatial variations. The increasing fuel split and the associated degree of inhomogeneity along the line from Case 1 to 3 manifests itself in increasing contrast of the spatially resolved auto-covariances. For values of $\tau<3 \mathrm{~ms}$, Case 1 and 2 show qualitatively less variations in spatially resolved autocovariances in comparison to Case 3 , where a clear separation in terms of shorter timescales can be identified in the shear layers between the wake of the pilot and both halves of the main stage. Figure 10 shows extracted line plots on a longer temporal axis of the auto-covariance for a more indepth comparison. Characteristic frequencies for Case 1 and 2 as identified by the FTs are also evident in the temperature auto-covariances. The higher peak prominence for the 


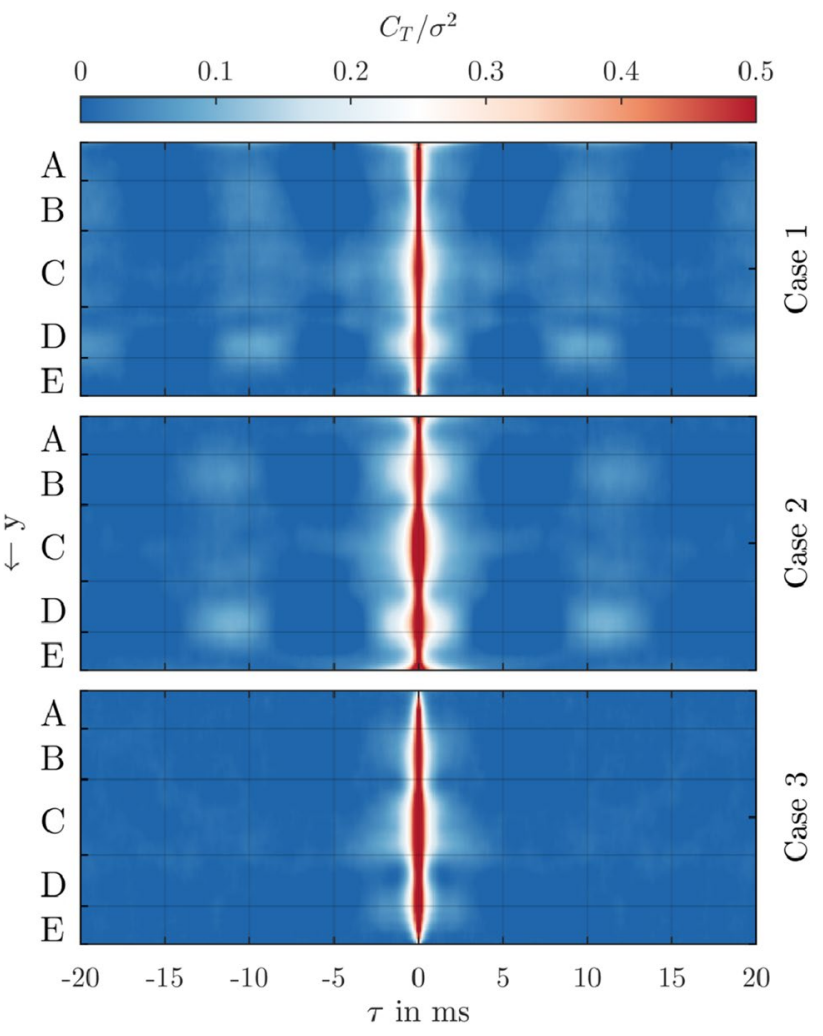

Fig. 9 Variance normalized temperature auto-covariances for all cases. Colorbar range is clipped at 0.5 to emphasize the tails of the auto-covariance

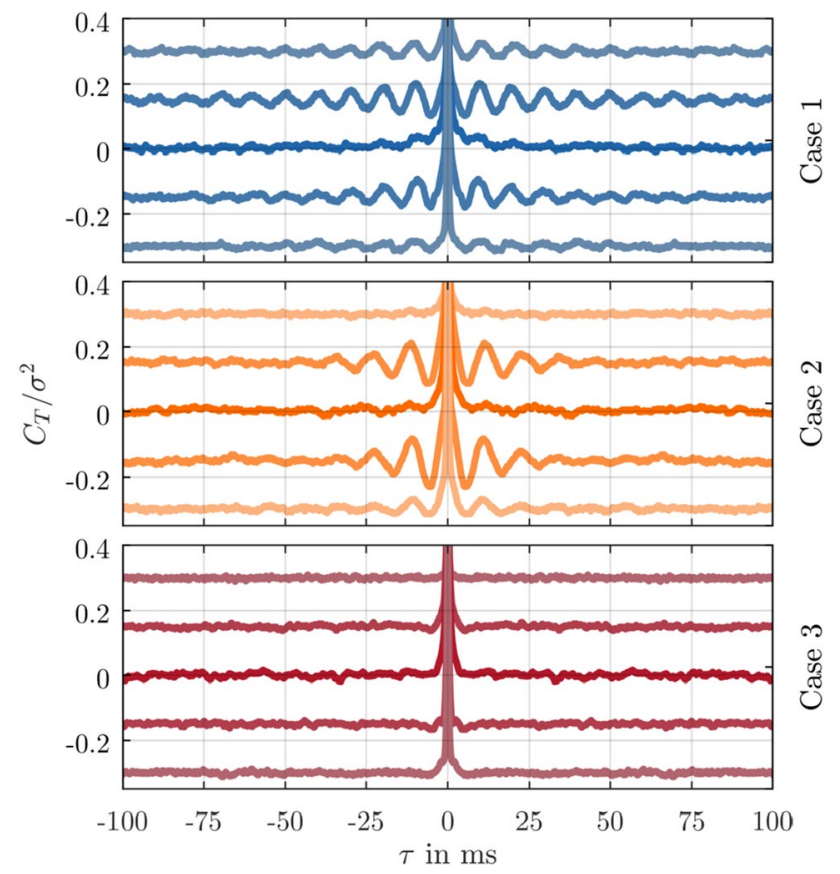

Fig. 10 Auto-covariance in different regions. Lines are intentionally shifted by 0.15 to visualize qualitative difference between the curves. From top to bottom with different color saturation: $\mathrm{A} \rightarrow \mathrm{E}$ characteristic frequency in Case 1 leads to slowly decreasing amplitudes with temporal lag $\tau$. In contrast, covariances decay more rapidly for Case 2, where the frequency peak is surrounded by strong broadband noise. Compared to Case 1 , the oscillations in auto-covariance are confined in a narrower spatial region and show a higher peak amplitude. A notable feature of the reconstructed oscillation is that along the nominal center line $(\mathrm{C})$, temperature stays almost constant over the entire period, see Fig. 8. This leads to long trailing auto-covariances without oscillations even though this is surrounded by high amplitude fluctuations in regions B and D, see Fig. 7. As expected, auto-covariances for Case 3 are free from oscillations.

To extract integral timescales from the auto-covariances, one has to account for the oscillations in the signal before integration. As for a highly oscillating case, covariances assume negative values but are by no means uncorrelated, a plain integration will underestimate the integral time scales in areas with significant amplitude. Thus, two methods were developed to account for this effect. The first method fits a sum of five exponentials to the data under the constraint that the fitted curve lies in between the peaks and valleys of the oscillations, i.e. the zero-crossings of the modulation. The second method uses the result of the first method but adds the envelope of the residual between the fit result and the data to the fit result. Figure 11 shows a comparison of the two proposed methods for two cases with varying influence from oscillations. Additionally to the benefit of taking the oscillations into account, this approach further

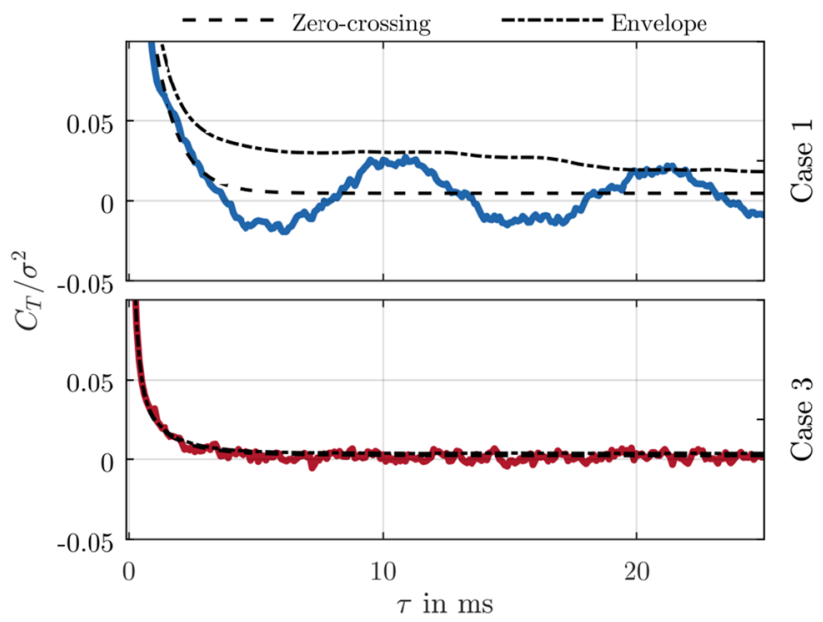

Fig. 11 Comparison of different methods to account for oscillations in the auto-covariance sequences. Top: exemplary auto-covariance sequence extracted from Case 1 with significant contribution from oscillations; bottom: exemplary profile from Case 3 without any oscillations present. For the oscillation-free case, both methods yield a good approximation of the auto-covariance. For the oscillating case, the envelope method amplifies computed integral line scales in area that are dominated by oscillations 

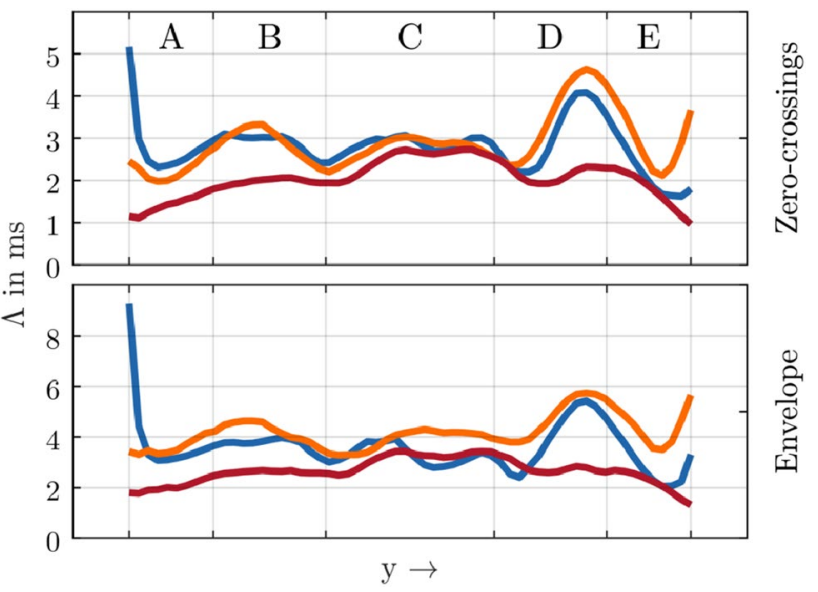

Fig. 12 Spatially resolved integral timescales. Top: Zero-crossing method; bottom: envelope method. Blue: Case 1; Orange: Case 2; red: Case 3

reduces the influence of noise in the evaluation. Extracted spatially resolved timescales using both methods are shown in Fig. 12. In comparison to the oscillation-free Case 3, Case 1 and 2 show significantly longer timescales in the wake of the main stage $(\mathrm{B}, \mathrm{D})$ as well as close to the walls $(\mathrm{A}, \mathrm{E})$. The timescales in region $\mathrm{C}$ seem to be dominated by the pilot, as there is no significant variation of integral timescales with varying fuel split. For Case 1, the longest timescales are found in region A, where the interaction between PVC and wall is assumed from the modal analysis. Overall, timescales are in the ms range, proving the $100 \mu$ s time separation between samples to be sufficient to resolve the phenomena at hand.

\subsection{Correlation analysis}

Analogously to the auto-correlation analysis, a cross-correlation between $\mathrm{OH}^{*}$ chemiluminescence in the primary zone, recorded with the PMT, and the temperature fluctuations in the exhaust can be evaluated. PMT data has been downsampled to $10 \mathrm{kHz}$ to match the temporal resolution of both measurement systems. Temporal synchronization was ensured by recording the IRO gate trigger along with the PMT signal. Figure 13 shows the resulting correlations for cases $1-3$. Consistent with the preceding analyses, the crosscorrelations for Case 3 are free from oscillations and feature a more impulse-response like behavior. A single correlation peak with only little spatial variation at a temporal lag of $\approx 4-7 \mathrm{~ms}$ can be identified. This lies within the expected range for convective transport from the primary zone to the exhaust region as estimated from PIV measurements at a similar configuration (Hassa et al. 2017). For Case 1 and 2 , the same characteristic V-shaped coherent patterns from the modal reconstruction are found in the cross-correlations

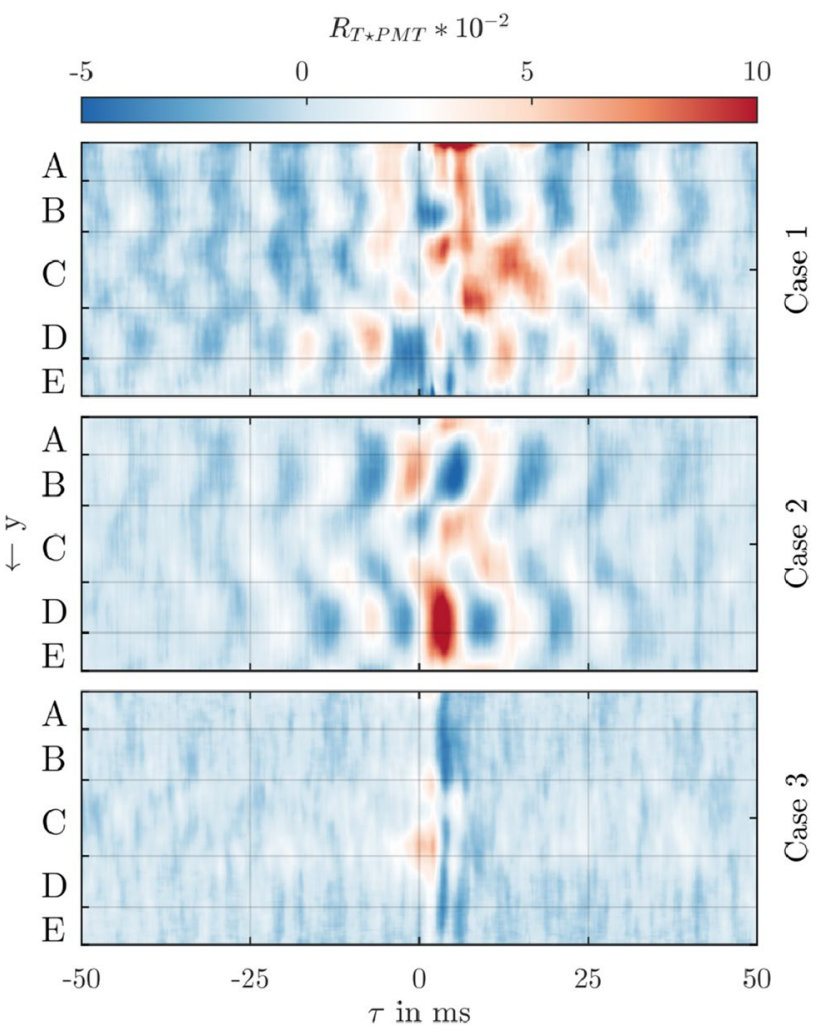

Fig. 13 Spatially resolved cross-correlation of $\mathrm{OH}^{*}$ with temperature profiles

in regions $\mathrm{B} \rightarrow \mathrm{C}$. For Case 1 , the location of the peak crosscorrelations is close to the wall (A), indicating that the periodic temperature drop has its origin already in the primary zone. Just as in the modal reconstruction, the characteristic pattern is more prominent for Case 2 due to stratification. These findings support the hypothesis that the oscillations are caused in the primary zone by a precessing vortex core. Oscillations of the left and right part of the main are $\approx 130^{\circ}$ phase shifted in both cases. Amplitudes in region B and D decay to $1 / e$ after about $60 \mathrm{~ms}$, i.e. $\approx 15-20$ integral timescales for Case 1 and $25 \mathrm{~ms}$, i.e. $\approx 4-5$ integral timescales for Case 2. Again, the peak prominence of the oscillation for Case 1 leads to a higher contrast in temporal direction while the stratification and the associated temperature inhomogeneity in Case 2 leads to a higher contrast in spatial direction.

\section{Conclusion}

Within this study, we presented spatially and temporally resolved temperature profile measurements in the exhaust of a single sector gas turbine combustor, operated at boundary conditions that are similar to the conditions found in aeroengines in flight. Additionally $\mathrm{OH}^{*}$ chemiluminescence in the primary zone was measured 
as an indicator for local heat release. Three different operating conditions with varying fuel split and air-tofuel ratio where investigated. With increasing fuel-split from Case 1 through Case 3, the mean temperature profile exhibits stronger inhomogeneities. Accordingly, areas of high fluctuations shift from close to the combustor walls towards the center of the combustor. The temporal resolution allowed for spatially resolved frequency analysis and modal reconstruction, revealing partly the phenomena responsible for the temperature fluctuations. For Case 1 (non-stratified, low AFR), temperature fluctuations close to the walls are likely to be induced by the precessing vortex core interacting with the wall. Case 2 (stratified, high AFR) shows a higher temperature amplitude resulting from the precessing vortex core due to fuel stratification. However, the influence is confined in the central region of the burner and does not interact with the wall. It has to be noted that with the available data from temperature and flow-field measurements (Willert et al. 2019), the presence of a precessing vortex core is very likely but still to be verified using additional instrumentation. Case 3 (pilot-only, high AFR) is free from prominent oscillations and exhibits a broadband noise frequency spectrum. Cross-correlations with $\mathrm{OH}^{*}$ chemiluminescence support the hypothesis of the precessing vortex core inducing the coherent patterns in the exhaust by linking the temperature profiles to events in the primary zone.

Acknowledgements Open Access funding provided by Projekt DEAL. The experiments presented herein were funded through the Clean Sky 2 Joint Undertaking Project CORNET (CORe Engine Noise Technology) under the European Union's Horizon 2020 research and innovation programme (grant agreement No 686332). The authors further would like to thank Thomas Soworka, Olaf Diers and Jan-Hendrik van Klaveren for their great support during the preparation and operation of the HBK1 test facility and OCORE 2 rig.

Funding Clean Sky 2 Joint Undertaking Project CORNET (CORe Engine Noise Technology) under the European Union's Horizon 2020 under grant agreement no 686332

Availability of data and material Due to the fact an industrial burner was used, data will not be made publicly available.Code availability A custom code was developed and used for all data processing presented in this manuscript.

\section{Compliance with ethical standards}

Conflict of Interest The authors declare that they have no conflict of interest.
Open Access This article is licensed under a Creative Commons Attribution 4.0 International License, which permits use, sharing, adaptation, distribution and reproduction in any medium or format, as long as you give appropriate credit to the original author(s) and the source, provide a link to the Creative Commons licence, and indicate if changes were made. The images or other third party material in this article are included in the article's Creative Commons licence, unless indicated otherwise in a credit line to the material. If material is not included in the article's Creative Commons licence and your intended use is not permitted by statutory regulation or exceeds the permitted use, you will need to obtain permission directly from the copyright holder. To view a copy of this licence, visit http://creativecommons.org/licenses/by/4.0/.

\section{References}

Arnold A, Bombach R, Käppeli B, Schlegel A (1997) Quantitative measurements of $\mathrm{OH}$ concentration fields by two-dimensional laser-induced fluorescence. Appl Phys B: Lasers Opt. https://doi. org/10.1007/s003400050218

Bake F, Richter C, Mühlbauer B, Kings N, Röhle I, Thiele F, Noll B (2009) The entropy wave generator (EWG): a reference case on entropy noise. J Sound Vib 326(3):574-598. https://doi. org/10.1016/j.jsv.2009.05.018. http://www.sciencedirect.com/ science/article/pii/S0022460X09004556

Bauerheim M, Duran I, Livebardon T, Wang G, Moreau S, Poinsot T (2016) Transmission and reflection of acoustic and entropy waves through a stator-rotor stage. J Sound Vib 374:260-278. https:// doi.org/10.1016/j.jsv.2016.03.041. http://www.sciencedirect.com/ science/article/pii/S0022460X16300165

Devillers R, Bruneaux G, Schulz C (2008) Development of a two-line $\mathrm{OH}$-laser-induced fluorescence thermometry diagnostics strategy for gas-phase temperature measurements in engines. Appl Opt 47(31):5871-5885. https://doi.org/10.1364/AO.47.005871

Doll U, Stockhausen G, Heinze J, Meier U, Hassa C, Bagchi I (2017) Temperature measurements at the outlet of a lean burn singlesector combustor by laser optical methods. J Eng Gas Turbines Power 139(2):21507. https://doi.org/10.1115/1.4034355

Doll U, Dues M, Bacci T, Picchi A, Stockhausen G, Willert C (2018) Aero-thermal flow characterization downstream of an NGV cascade by five-hole probe and filtered Rayleigh scattering measurements. Exp Fluids 59(10):150. https://doi.org/10.1007/s0034 8-018-2607-z

Giezendanner-Thoben R, Meier U, Meier W, Aigner M (2005) Phaselocked temperature measurements by two-line OH PLIF thermometry of a self-excited combustion instability in a gas turbine model combustor. Flow Turbul Combust 75(1-4):317-333. https://doi. org/10.1007/s10494-005-8587-0

Hassa C, Meier U, Heinze J, Magens E, Schroll M, Bagchi I (2017) Investigation of the reacting flow field of a lean burn injector with varying degree of Swirl at elevated pressure condition. In: ASME Turbo Expo 2017: turbomachinery technical conference and exposition, International Gas Turbine Institute, ASME, Charlotte, North Carolina, USA, vol 4B: Combustion, Fuels and Emissions, pp GT2017-64280, V04BT04A006. https://doi.org/10.1115/ GT2017-64280

Heinze J, Meier U, Behrendt T, Willert C, Geigle KP, Lammel O, Lückerath R (2011) PLIF thermometry based on measurements of absolute concentrations of the $\mathrm{OH}$ radical. Zeitschrift für Physikalische Chemie 225(11-12):1315-1341. https://doi.org/10.1524/ zpch.2011.0168

Hield PA, Brear MJ, Jin SH (2009) Thermoacoustic limit cycles in a premixed laboratory combustor with open and choked exits. Combust Flame 156(9):1683-1697. https://doi.org/10.1016/j.combu 
stflame.2009.05.011. http://www.sciencedirect.com/science/artic le/pii/S0010218009001576

Ihme M (2017) Combustion and engine-core noise. Annu Rev Fluid Mech 49:277-310

Luque J, Crosley D (1999) LIFBASE: database and simulation program (v 2.1.1). SRI International Report MP pp 99-009. https://www. sri.com/engage/products-solutions/lifbase

Meier U, Lange L, Heinze J, Hassa C, Sadig S, Luff D (2015) Optical methods for studies of self-excited oscillations and the effect of dampers in a high pressure single sector combustor. J Eng Gas Turbines Power 137(7)

Morgans AS, Duran I (2016) Entropy noise: a review of theory, progress and challenges. Int J Spray Combust Dyn 8(4):285-298

Morley C (2005) GASEQ: a chemical equilibrium program for windows. Version 079b http://www.gaseq.co.uk

Pixner P, Schiessl R, Dreizler A, Maas U (2000) Experimental determination of Pdfs of $\mathrm{OH}$ radicals in IC engines using calibrated laserinduced fluorescence as a basis for modelling the end-phase of engine combustion. Combust Sci Technol 158(1):485-509. https ://doi.org/10.1080/00102200008947346
Sadanandan R, Meier W, Heinze J (2012) Experimental study of signal trapping of $\mathrm{OH}$ laser induced fluorescence and chemiluminescence in flames. Appl Phys B 106(3):717-724. https://doi. org/10.1007/s00340-011-4704-z

Weber V, Brübach J, Gordon R, Dreizler A (2011) Pixel-based characterisation of CMOS high-speed camera systems. Appl Phys B 103(2):421-433. https://doi.org/10.1007/s00340-011-4443-1

Willert C, Schroll M, Heinze J, Soworka T (2019) High-speed PIV at the exit of a lean-burn combustion chamber operated at elevated pressure. In: Kähler CJ, Hain R, Scharnowski S, Fuchs T (eds) Proceedings of the 13th international symposium on particle image velocimetry. https://doi.org/10.18726/2019_3

Publisher's Note Springer Nature remains neutral with regard to jurisdictional claims in published maps and institutional affiliations. 